\title{
A phase II trial of capecitabine plus cisplatin (XP) for patients with advanced gastric cancer with early relapse after S-1 adjuvant therapy: XParTS-I trial
}

\author{
Kazuhiro Nishikawa' ${ }^{1}$ - Akira Tsuburaya ${ }^{2} \cdot$ Takaki Yoshikawa $^{3} \cdot$ Masazumi Takahashi $^{4} \cdot$ Kazuaki Tanabe $^{5}$. \\ Kensei Yamaguchi ${ }^{6}$. Shigefumi Yoshino ${ }^{7}$. Tsutomu Namikawa ${ }^{8} \cdot$ Toru Aoyama $^{3}$. Yasushi Rino ${ }^{9}$. Junji Kawada ${ }^{10}$. \\ Akihito Tsuji ${ }^{11}$. Koichi Taira ${ }^{12}$. Yutaka Kimura ${ }^{13}$. Yasuhiro Kodera ${ }^{14}$. Yoshinori Hirashima ${ }^{15}$. Hiroshi Yabusaki ${ }^{16}$. \\ Naoki Hirabayashi ${ }^{17} \cdot$ Kazumasa Fujitani $^{10} \cdot$ Yumi Miyashita ${ }^{18}$. Satoshi Morita ${ }^{19}$. Junichi Sakamoto ${ }^{20}$
}

Received: 18 November 2017 / Accepted: 19 February 2018 / Published online: 27 February 2018

(c) The International Gastric Cancer Association and The Japanese Gastric Cancer Association 2018

\begin{abstract}
Backgrounds In Japan, standard regimens for advanced gastric cancer (AGC) include S-1 chemotherapy. The standard treatment for early relapse after adjuvant chemotherapy with fluoropyrimidine alone is platinum-based chemotherapy, while the standard treatment for early relapse after adjuvant chemotherapy with fluoropyrimidine plus platinum is second-line chemotherapy. To evaluate the efficacy and safety of capecitabine plus cisplatin (XP) treatment for AGC patients who relapse within 6 months after S-1-based therapy, we conducted a multicenter phase II trial (NCT01412294).

Methods HER2-negative gastric cancer patients treated with adjuvant chemotherapy including S-1 for more than 12 weeks and relapsed within 6 months were treated with capecitabine $1000 \mathrm{mg} / \mathrm{m}^{2}$ bid for 14 days plus cisplatin $80 \mathrm{mg} / \mathrm{m}^{2}$ on day 1 of a 3-week cycle. The primary endpoint was PFS; secondary endpoints were OS, time to treatment failure, overall response rate (ORR) and safety.

Results Forty patients (median age 64) were enrolled; of those, 37 (92.5\%) received adjuvant S-1 monotherapy. Median PFS was 4.4 months (95\% CI 3.6-5.1), which was longer than the 2-month protocol-specified threshold $(p<0.001)$. Median OS was 13.7 months (95\% CI 9.0-17.7) and ORR was 8/30 (26.7\%) (95\% CI 14.2-44.4). Most common grade $\geq 3$ adverse events were neutropenia (23\%), anemia (18\%), elevated serum creatinine (18\%), fatigue (13\%), diarrhea (7.5\%), and anorexia (7.5\%). Conclusions XP was safe and effective in patients with early relapse after S-1 adjuvant chemotherapy for curatively resected gastric cancers. XP may be a good option for the treatment of patients after early failure after adjuvant S-1.
\end{abstract}

Trial registration NCT01412294.

Keywords Advanced gastric cancer · Capecitabine plus cisplatin (XP) · S-1 adjuvant therapy $\cdot$ Early relapse ·

Fluoropyrimidine switching

\section{Introduction}

Gastric cancer is the third leading cause of death worldwide [1]. S-1 monotherapy has been considered the standard adjuvant treatment after curative gastrectomy of stage II or III gastric cancer patients in Japan [2]. However, almost half of those patients eventually experience disease relapse, and their long-term prognosis is unfavorable [3]. After the results

Kazuhiro Nishikawa

kazuno1@onh.go.jp; kazuno13@hotmail.co.jp

Extended author information available on the last page of the article were reported from the JCOG9912 and SPIRITS trials, S-1 plus cisplatin (SP) became accepted as the standard treatment for patients with advanced gastric cancer (AGC) in Japan $[4,5]$. In a retrospective analysis of those patients by Shitara et al., SP was not shown to be effective in patients with recurrent gastric cancer, especially with a relapse-free interval (RFI) of less than 6 months after adjuvant S- 1 chemotherapy [6]. In their report, compared with patients with RFI of $\geq 6$ months, patients with RFI of $<6$ months had a significantly lower objective overall response rate (ORR) (5.0 vs. $37.5 \%$ ), shorter progression-free survival (PFS) (2.3 vs. 6.2 months), and shorter overall survival (OS) (7.3 vs. 16.6 months). In this regard, establishment of effective 
treatment options for early relapse after S-1 adjuvant chemotherapy is required. Although no prospective study has evaluated chemotherapy specifically for patients who have failed adjuvant $\mathrm{S}-1$, a phase II study of capecitabine plus cisplatin (XP) in 32 patients with gastric cancer that recurred within or more than 6 months after adjuvant chemotherapy with doxifluridine or 5-fluorouracil (5-FU)-containing regimens was reported [7]. In this trial, response rates (39 vs. $21 \%, p=0.427)$, time to treatment failure (TTF) $(8.3$ vs. 5.4 months, $p=0.072$ ), and OS (14.1 vs. 9.3 months, $p=0.075)$ tended to be better in patients with an RFI of $>6$ months $(n=13)$ than in patients with an RFI $<6$ months $(n=19)$, although the differences did not reach statistical significance.

As to the first-line setting, combined administration of fluoropyrimidine and a platinum is the standard chemotherapy for advanced or recurrent gastric cancer, while triplet chemotherapy using docetaxel, cisplatin, and fluorouracil; epirubicin, cisplatin, and fluorouracil; or epirubicin, oxaliplatin, and capecitabine, are other treatment options $[8$, 9]. In Japan, SP is the most prevalent combination chemotherapy for first-line treatment, as above [5]. Kang and colleagues evaluated the non-inferiority of XP compared with 5-FU plus cisplatin. The median progression-free survival (PFS) showed significant non-inferiority (5.6 months vs. 5.0 months; $\mathrm{HR}=0.81,95 \%$ confidence interval (CI) $0.63-1.04, p<0.001$ ) [10]. Based on these results, XP could now be considered one of the standard treatments for AGC [11], and XP was adopted as the reference arm in two recent global studies of molecular targeted agents [12, 13]. However, the usefulness of XP for early relapse after S-1 adjuvant therapy has not yet been assessed.

In this regard, the objectives of this study were to estimate the efficacy and safety of XP in patients with AGC who relapsed within 6 months after S-1-based adjuvant therapy.

\section{Patients and methods}

\section{Eligibility}

Eligibility requirements included: patients who had received adjuvant chemotherapy for gastric cancer including S-1 for more than 12 weeks, and relapsed within 6 months thereafter; were aged 20-74 years at the time of informed consent; were HER 2 negative; and had histologically confirmed gastric adenocarcinoma. Other inclusion criteria were as follows: lesions confirmed by imaging no more than 28 days before registration [not required for measurable lesions as defined in Response Evaluation Criteria In Solid Tumors (RECIST) version 1.1]; no previous chemotherapy or radiotherapy except for S-1-based adjuvant chemotherapy; last dose of anticancer drug in the prior chemotherapy $\geq 14$ days before enrollment; Eastern Cooperative Oncology Group performance status (PS) of 0-2; life expectancy of at least 3 months after registration; and adequate organ function [neutrophil count $\geq 1500 / \mathrm{mm}^{3}$, hemoglobin $\geq 9.0 \mathrm{~g} / \mathrm{dL}$, aspartate aminotransferase (AST), alanine aminotransferase $($ ALT) $\leq 2.5 \times$ upper limit of normal (ULN) in each institution ( $\leq 5$ times in cases of metastases to liver), ALP $\leq 2.5 \times$ ULN in each institution $(\leq 5$ times in cases of metastases to liver, and $\leq 10$ times in cases of metastases to bone), total bilirubin $\leq 1.5 \times \mathrm{ULN}$ in each institution, and creatinine clearance $\geq 60 \mathrm{~mL} / \mathrm{min}$ (as estimated using the Cockcroft-Gault equation)]. Exclusion criteria included: active second primary malignancy; severe or uncontrolled concurrent disease; overt infection or inflammation; psychiatric disorder being treated or requiring an antipsychotic therapy; active hepatitis; previous treatment with cisplatin of more than a total dose of $120 \mathrm{mg} / \mathrm{m}^{2}$; previous history of serious hypersensitivity to fluoropyrimidines or platinum agents; previous history of adverse reactions suggestive of dihydropyrimidine dehydrogenase (DPD) deficiency; being treated or in need of treatment with flucytosine, phenytoin, or warfarin potassium; chronic diarrhea (watery stools or stools $\geq 4$ times/day); active gastrointestinal bleeding; pericardial effusion, or pleural effusion or ascites requiring drainage; unwillingness to practice contraception; poor oral intake; pregnant or lactating females, or females wishing to become pregnant; and otherwise determined by investigators or site principal investigators to be unsuitable for participation in the study. All eligible patients provided written informed consent before enrollment.

\section{Treatment}

Within 14 days after enrollment, eligible patients started treatment with XP on a 3-week cycle. Capecitabine was administered orally at a dose of $1000 \mathrm{mg} / \mathrm{m}^{2}$ twice daily (equivalent to a total daily dose of $2000 \mathrm{mg} / \mathrm{m}^{2}$ ) for 2 weeks (day 1-14). Cisplatin $80 \mathrm{mg} / \mathrm{m}^{2}$ on day 1 of each cycle was given by intravenous infusion over $2 \mathrm{~h}$. Treatments were discontinued at the following events: progressive disease (PD) based on RECIST version 1.1 or clinically determined progression of the primary disease; conversion to resectable disease; an unacceptable adverse event; patient refusal to continue treatment; and death. If treatment continuation with cisplatin was determined to be unfeasible before any progression was confirmed, continuous monotherapy with capecitabine was continued until PD.

\section{Evaluation}

At baseline, patient general characteristics and medical history were reviewed, including diagnosis and macroscopic/ histologic classification of gastric cancer, imaging to identify 
measurable lesions, assessment of subjective and objective symptoms, and laboratory tests.

Tumor responses were classified using RECIST version 1.1 as complete response (CR), partial response (PR), stable disease (SD), or PD, and confirmed by investigators.

Adverse events were evaluated using the CTCAE version 4.0. Contrast-enhanced computed tomography or magnetic resonance imaging and plain chest X-rays were performed within 28 days before enrollment and repeated every 4 weeks after starting treatment, or every 6 weeks from week 16 onwards. During treatment, laboratory tests (hematology and blood chemistry) were performed on the day before every drug administration.

\section{Statistical methods}

This was a phase II, open-label, multicenter trial performed at 15 institutions across Japan. The primary endpoint was PFS. Secondary endpoints were OS, time to treatment failure (TTF), ORR, and the incidence and severity of adverse events.

Because the PFS was 2.3 months in recurrent gastric cancer with RFI of less than 6 months after adjuvant S-1 chemotherapy in the previous retrospective analysis [6], the threshold PFS was set at 2 months. The addition of XP was expected to increase PFS by 1 month. At a one-sided significance of $5 \%$ and a power of $80 \%, 37$ patients were required for this study. When ineligible patients or dropouts were included, the target sample size was estimated to be 40. All clinical data were held centrally at the ECRIN data center and analyzed using SAS for Windows version 9.3 (SAS Institute Inc., Cary, NC, USA).

This trial was conducted in compliance with the ethical principles of the Declaration of Helsinki and the Ethical Guidelines for Clinical Studies of the Japanese Ministry of Health, Labour and Welfare. This trial was approved by institutional review boards or ethics committees at all participating centers. This trial was registered with ClinicalTrials.gov, number NCT 01412294.

\section{Results}

\section{Patient characteristics}

Between June 2011 and April 2014, 40 patients were enrolled from 15 institutions in Japan. All patients were included in the final analyses. Median follow-up was 13.7 months (range 0.2-41.9 months). Patient characteristics are summarized in Table 1. Median age was 64, 32 males (80\%); adjuvant chemotherapy: regimen, S-1 monotherapy $(N=37,92.5 \%)$.
Table 1 Patient characteristics

\begin{tabular}{|c|c|c|}
\hline & $N$ & $\%$ \\
\hline \multicolumn{3}{|l|}{ Age, years } \\
\hline Median (range) & $65.5(43-77)$ & \\
\hline \multicolumn{3}{|l|}{ Sex } \\
\hline Male & 32 & 80 \\
\hline Female & 8 & 20 \\
\hline \multicolumn{3}{|l|}{ ECOG PS } \\
\hline 0 & 32 & 80 \\
\hline 1 & 7 & 17.5 \\
\hline 2 & 1 & 2.5 \\
\hline \multicolumn{3}{|l|}{ Relapse site } \\
\hline Local & 1 & 1.9 \\
\hline Lymph node & 15 & 27.8 \\
\hline Peritoneum & 18 & 33.3 \\
\hline Liver & 11 & 20.4 \\
\hline Lung & 2 & 3.7 \\
\hline Bone & 2 & 3.7 \\
\hline Other & 5 & 9.3 \\
\hline \multicolumn{3}{|l|}{ Adjuvant regimen } \\
\hline S-1 & 37 & 92.5 \\
\hline S-1 + taxane & 3 & 7.5 \\
\hline \multicolumn{3}{|l|}{ Adjuvant courses } \\
\hline Average (standard deviation) & $7.1(3.2)$ & \\
\hline Median (range) & $7.0(3-18)$ & \\
\hline \multicolumn{3}{|l|}{ Completion of adjuvant } \\
\hline Completed & 11 & 27.5 \\
\hline Discontinued & 29 & 72.5 \\
\hline
\end{tabular}

ECOG Eastern Cooperative Oncology Group, PS performance status

\section{Treatments}

The median number of cycles of XP was 5 (1-27). Twentytwo patients (55\%) required dose reduction of capecitabine and 26 patients $(65 \%)$ required dose reduction of cisplatin. The dose intensity for all treatment cycles was $79 \%$ for capecitabine, and that for the first six cycles was $62 \%$ for cisplatin.

At the time of final analysis, seven patients continued on protocol treatment. Twenty-six patients received subsequent treatments (26/33: 78.8\%) following protocol treatment.

\section{Tumor responses}

Of the 30 patients with measurable lesions, one achieved CR, seven achieved PR, and 11 achieved SD. Therefore, the ORR (CR plus PR) was $26.7 \%$ (95\% CI $14.2-44.4 \%$; $N=8 / 30$ ), and the disease control rate (DCR) (CR plus PR plus SD) was $63.3 \%$ (95\% CI $45.5-78.1 \% ; N=19 / 30)$ (Table 2). Maximum \% changes in tumor measurements 
Table 2 Response rate

\begin{tabular}{lrr}
\hline$N=30$ & $N$ & $95 \% \mathrm{CI}$ \\
\hline $\mathrm{CR}$ & $1(3.3)$ & \\
$\mathrm{PR}$ & $7(23.3)$ & \\
$\mathrm{SD}$ & $11(36.7)$ & \\
$\mathrm{PD}$ & $4(13.3)$ & \\
NE & $7(23.3)$ & $14.2-44.4$ \\
RR (CR, PR) & $8(26.7)$ & $45.5-78.1$ \\
DCR (CR, PR, SD) & $19(63.3)$ &
\end{tabular}

$C R$ complete response, $P R$ partial response, $S D$ stable disease, $P D$ progressive disease, $N E$ not evaluable, $R R$ response rate, $D C R$ disease control rate

from baseline according to best overall response are shown in Fig. 1.

\section{Survival}

The median PFS was 4.4 months (95\% CI 3.6-5.1), which was longer than the protocol-specified threshold of 2 months $(p<0.001)$ (Fig. 2a). Median OS was 13.7 months (95\% CI 9.0-17.7) (Fig. 2b) and median TTF was 4.0 months (95\% CI 3.1-5.1).

\section{Adverse events}

The incidence of grade $3 / 4$ adverse events was low (Table 3 ). There was no event of clinically significant cumulative toxicity. There was no death resulting from toxicities. The most common grade $3 / 4$ adverse events were neutropenia in nine patients (22.5\%), anemia in seven patients (17.5\%), hyponatremia in five patients (12.5\%), fatigue in five patients $(12.5 \%)$, diarrhea in three patients $(7.5 \%)$, and anorexia in three patients $(7.5 \%)$. Other grade $3 / 4$ toxicities were

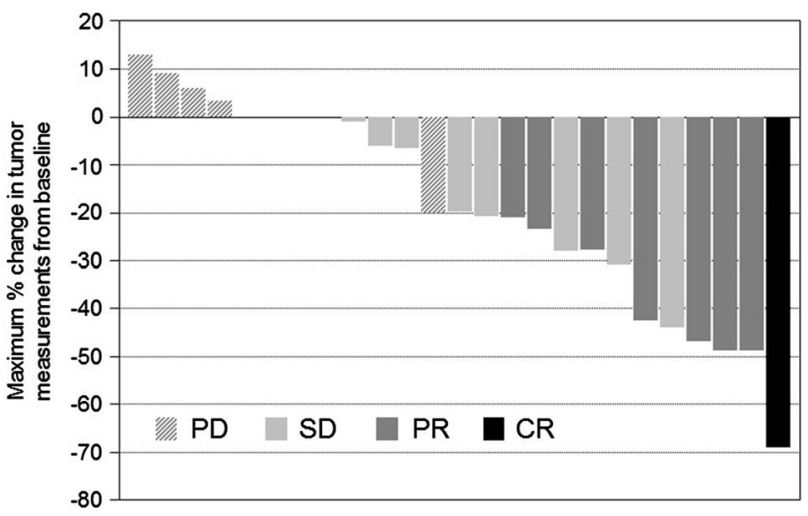

Fig. 1 Waterfall plot of the best overall response in individual patients. $C R$ complete response, $P R$ partial response, $S D$ stable disease, $P D$ progressive disease
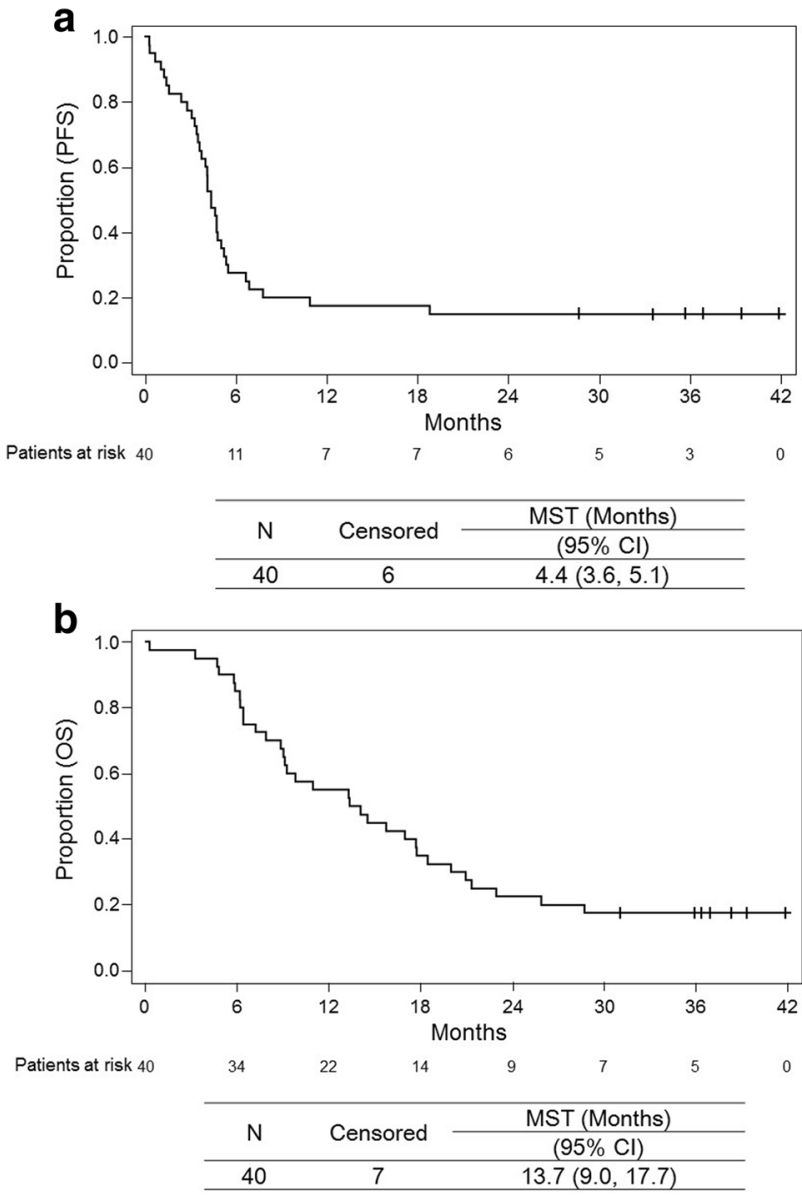

Fig. 2 Kaplan-Meier plots of progression-free survival (a), and overall survival (b). $C I$ confidence interval, $M S T$ median survival time, $P F S$ progression-free survival, $O S$ overall survival

thrombocytopenia and febrile neutropenia that occurred in two patients $(5.0 \%)$, and nausea, oral mucositis, and hand-foot syndrome that occurred in one patient (2.5\%).

\section{Discussion}

This is the first report of a phase II study examining the efficacy and safety of XP in patients with AGC, who relapsed within 6 months after S-1-containing adjuvant chemotherapy. XP-treated patients achieved favorable PFS, OS, and ORR compared with SP in this setting [6]. This combination therapy was also tolerable, with a low rate of grade $3 / 4$ adverse events; most adverse events were of grade $1 / 2$. The primary endpoint was met because the median PFS was 4.4 months (95\% CI 3.6-5.1) $(p<0.001)$.

It was interesting to evaluate the effects of re-challenge with different types of fluoropyrimidines after the failure of another drug, although fluoropyrimidine switching is still controversial $[14,15]$. The response rate of $26.7 \%$ in the 
Table 3 Adverse events $(N=40)$

\begin{tabular}{llllll}
\hline & \multicolumn{2}{l}{ Grades $1-4$} & & \multicolumn{2}{l}{ Grade $3 / 4$} \\
\cline { 2 - 3 } & $N$ & $\%$ & & $N$ & $\%$ \\
\hline Neutropenia & 26 & 65.0 & & 9 & 22.5 \\
Anemia & 36 & 90.0 & & 7 & 17.5 \\
Thrombocytopenia & 26 & 65.0 & & 2 & 5.0 \\
Serum AST value increased & 16 & 40.0 & & 0 & 0.0 \\
Serum ALT value increased & 13 & 32.5 & & 0 & 0.0 \\
Serum total bilirubin value increased & 5 & 12.5 & & 0 & 0.0 \\
Serum creatinine value increased & 19 & 47.5 & & 0 & 0.0 \\
Hypoalbuminemia & 24 & 60.0 & & 0 & 0.0 \\
Hyponatremia & 25 & 62.5 & & 5 & 12.5 \\
Hyperkalemia & 18 & 45.0 & & 0 & 0.0 \\
Febrile neutropenia & 2 & 5.0 & & 2 & 5.0 \\
Fatigue & 21 & 52.5 & & 5 & 12.5 \\
Fever & 6 & 15.0 & & 0 & 0.0 \\
Weight loss & 12 & 30.0 & & 0 & 0.0 \\
Anorexia & 31 & 77.5 & & 3 & 7.5 \\
Nausea & 23 & 57.5 & & 1 & 2.5 \\
Vomiting & 9 & 22.5 & & 0 & 0.0 \\
Oral mucositis & 15 & 37.5 & & 1 & 2.5 \\
Diarrhea & 11 & 27.5 & & 3 & 7.5 \\
Allergen reaction & 1 & 2.5 & & 0 & 0.0 \\
Peripheral sensory neuropathy & & 25.0 & & 0 & 0.0 \\
Hand-foot syndrome & & 40.0 & & 1 & 2.5 \\
\hline
\end{tabular}

$A S T$ aspartate aminotransferase, $A L T$ alanine aminotransferase

present study was comparable with that previously reported by Kang et al. for XP after adjuvant chemotherapy (21\%) [7]. One possible reason for the high response rate observed in patients with early recurrence of GC is that we did not use the same fluoropyrimidine (capecitabine after S-1 versus capecitabine after doxifluridine or 5-FU) as Kang et al.

$\mathrm{S}-1$ is an oral anticancer drug composed of the $5-\mathrm{FU}$ prodrug, tegafur, and two 5-FU modulators; it has achieved high response rates in patients with gastric cancer in phase II studies [16, 17]. Capecitabine is also an oral fluoropyrimidine that is metabolized primarily in the liver and converted in tumor tissues to 5-FU by the enzyme thymidine phosphorylase (TP), which is found in higher concentrations in tumor than in normal cells [18]. These two types of oral fluoropyrimidines show some different characteristics in the mechanisms of their antitumor effect. A subset analysis of the FLAGS trial showed that S-1 seemed to be better than 5 -FU in the subgroup with diffuse-type gastric cancer [19]. This result was consistent with the results of a subset analysis of the JCOG9912 trial, which showed that S-1 was better than 5-FU in patients with diffuse-type gastric cancer or with gastric cancer associated with high dihydropyrimidine dehydrogenase (DPD), with diffuse-type tumors associated more commonly than intestinal types with high DPD [20]. This result was expected, since $\mathrm{S}-1$ consists of tegafur, otastat potassium, and gimestat, a potent competitive inhibitor of DPD. Capecitabine is transformed to 5-FU in several steps, with the final step involving TP, as above [17]. A phase II trial in Japan showed that the response rate (RR) was significantly higher (Fisher's exact test, $p=0.028$ ) in patients with TP-positive and DPD-negative tumors $(60 \%, 6 / 10)$ than in the remaining patients $(13 \%, 2 / 15)$ [21]. In contrast, high expression of TP was reported to be negatively associated with the efficacy of 5-FU or S-1 in gastric cancer [22, 23].

Conventional exclusion criteria in clinical trials in the first-line setting omitted patients who experienced disease recurrence within 6 months after the last adjuvant chemotherapy $[4,5,10,13]$. The standard treatment for early relapse after adjuvant chemotherapy with fluoropyrimidine alone is platinum-based chemotherapy, such as XP, XELOX and FOLFOX. However, the usefulness of platinum-based chemotherapy in this setting has not been reported much. Besides this, these early relapsed patients after adjuvant therapy sometimes used second-line chemotherapy regimens such as irinotecan, taxane, or irinotecan plus cisplatin [24-26]. These prior reports evaluated other second-line chemotherapies and reported median PFS periods of 2.3-3.8 months, median OS periods of 5.2-10.7 months, and ORRs of 7-22\% [24-26]. In contrast, median PFS was 4.4 months (95\% CI 3.6-5.1), median OS was 13.7 months (95\% CI 9.0-17.7), and ORR was $26.7 \%$ (95\% CI 14.2-44.4\%; $N=8 / 30$ ) in our study. The efficacy of XP compared with other chemotherapies (irinotecan, taxane or irinotecan plus cisplatin) for recurrence after adjuvant S-1 should be evaluated in future clinical trials.

There are two possible explanations for these longer PFS and OS and high ORR. First, all patients in our study received the protocol treatment without prior use of platinum, which resulted in a favorable PFS of 4.4 months and an ORR of $26.7 \%$. Second, the proportion of patients who received subsequent chemotherapy after disease progression (following therapy) was high (26/33 patients, $78.8 \%$ ). The subsequent therapy might also contribute to the large difference between OS and PFS in this study (13.7 and 4.4 months, respectively).

The incidence of grade 3/4 adverse events in this study was similar to that in an earlier study using the same XP regimen for patients who recurred after adjuvant chemotherapy with doxifluridine or 5-FU-containing regimens. In that study, neutropenia (38\%), leukopenia (9\%), anemia $(16 \%)$, diarrhea $(5 \%)$, anorexia $(2 \%)$, and peripheral sensory neuropathy $(2 \%)$ were the most common grade $3 / 4$ adverse events in the cohort of 32 patients treated with XP [7]. In our study of 40 patients, neutropenia (22.5\%), anemia (17.5\%), hyponatremia (12.5\%), fatigue (12.5\%), diarrhea $(7.5 \%)$, and anorexia (7.5\%) were the most common grade $3 / 4$ adverse events. These results suggest that XP 
was well tolerated in patients with recurrent gastric cancer after fluoropyrimidine-based adjuvant chemotherapy.

However, there are some limitations of this study. First, because this was not a randomized comparative study, the selected population may have been biased toward patients with good performance status (PS) and low tumor burden. Second, the moderate sample size in a single-country study was another limitation. The inclusion of only Japanese patients may also limit the generalizability of this study. Third, the efficacy of XP compared with other chemotherapies (irinotecan, taxane and/or ramucirumab) for recurrence after adjuvant $S-1$ should be evaluated in future randomized trials.

In conclusion, $\mathrm{XP}$ was generally well tolerated and effective in patients with AGC who relapsed within 6 months after S-1-based adjuvant therapy. XP may be a good option for this cohort of patients in Japan.

Acknowledgements We thank the investigators from the following institutions who enrolled patients for this trial: Osaka General Medical Center, Yokohama Municipal Citizen's Hospital, Hiroshima University Graduate School of Medicine, Saitama Cancer Center, Yokohama City University, Yamaguchi University Graduate School of Medicine, Kochi Medical School, Kanagawa Cancer Center, Yokohama City University, Kobe City Medical Center General Hospital, Osaka City General Hospital, Sakai Municipal Hospital, Nagoya University Graduate School of Medicine, Oita University Faculty of Medicine, Hiroshima City Asa Hospital, and Niigata Cancer Center Hospital. Furthermore, we deeply appreciate all patients who participated in the trial. We acknowledge Dr Mark Abramovitz of Edanz Medical Writing for editorial support, which was funded by the non-profit organization Epidemiological and Clinical Research Information Network (ECRIN).

Funding This work was supported by the non-profit organization Epidemiological and Clinical Research Information Network (ECRIN).

\section{Compliance with ethical standards}

Conflict of interest The individual conflict of interest disclosure is as follows: K. Nishikawa has received honoraria from Chugai, Taiho, Yakult, Eli Lilly, Tsumura, and EA Pharma, and research funding from Yakult and Taiho, outside the submitted work. T. Yoshikawa has received lecture fees from Chugai, Taiho, Yakult, Eli Lilly and Ono, and for advisory work from Ono and MSD, outside the submitted work. K. Yamaguchi has received personal fees from Chugai, Taiho, Yakult, Takeda, Merck and Eli Lilly, and research funding from MSD, Merck, Bristol, Ono, Dainippon Sumitomo, Taiho, Daiichi-Sankyo and Yakult, outside the submitted work. S. Yoshino has received honoraria from Chugai, Taiho, Ono and Eli Lilly, outside the submitted work. Y. Kodera has received research funding from Chugai, Taiho, Daiichi-Sankyo, Bristol-Myers, Eli Lilly, Otsuka, Takeda, Yakult, CSL Behring, Pfizer, Ono, Kaken, Tsumura, EA Pharma, Novartis, KCI and the Japan Blood Products Organization, outside the submitted work. S. Morita has received honoraria from Chugai and Taiho, outside the submitted work. J. Sakamoto has received consultant fee from Takeda, and Honoraria from Tsumura and Chugai, outside the submitted work. None of the remaining authors have potential conflicts of interest to declare.
Ethical statements This trial was conducted in compliance with the ethical principles of the Declaration of Helsinki and the Ethical Guidelines for Clinical Studies of the Japanese Ministry of Health, Labour and Welfare. This trial was approved by the institutional review boards or ethics committees at all participating centers. This trial was registered with ClinicalTrials.gov (NCT01412294).

\section{References}

1. Ferlay J, Soerjomataram I, Dikshit R, Eser S, Mathers C, Rebelo $\mathrm{M}$, et al. Cancer incidence and mortality worldwide: sources, methods and major patterns in GLOBOCAN 2012. Int J Cancer. 2015;136:E359-86.

2. Sakuramoto S, Sasako M, Yamaguchi T, Kinoshita T, Fujii M, Nashimoto A, et al. Adjuvant chemotherapy for gastric cancer with S-1, an oral fluoropyrimidine. N Engl J Med. 2007;357:1810-20.

3. Sasako M, Sakuramoto S, Katai H, Kinoshita T, Furukawa $\mathrm{H}$, Yamaguchi T, et al. Five-year outcomes of a randomized phase III trial comparing adjuvant chemotherapy with S-1 versus surgery alone in stage II or III gastric cancer. J Clin Oncol. 2011;29:4387-93.

4. Boku N, Yamamoto S, Fukuda H, Shirao K, Doi T, Sawaki A, et al. Fluorouracil versus combination of irinotecan plus cisplatin versus S-1 in metastatic gastric cancer: a randomised phase 3 study. Lancet Oncol. 2009;10:1063-9.

5. Koizumi W, Narahara H, Hara T, Takagane A, Akiya T, Takagi M, et al. S-1 plus cisplatin versus S-1 alone for first-line treatment of advanced gastric cancer (SPIRITS trial): a phase III trial. Lancet Oncol. 2008;9:215-21.

6. Shitara K, Morita S, Fujitani K, Kadowaki S, Takiguchi N, Hirabayashi N, et al. Combination chemotherapy with S-1 plus cisplatin for gastric cancer that recurs after adjuvant chemotherapy with S-1: multi-institutional retrospective analysis. Gastric Cancer. 2012;15:245-51.

7. Kang HJ, Chang HM, Kim TW, Ryu MH, Sohn HJ, Yook JH, et al. Phase II study of capecitabine and cisplatin as first-line combination therapy in patients with gastric cancer recurrent after fluoropyrimidine-based adjuvant chemotherapy. Br J Cancer. 2005;92:246-51.

8. Van Cutsem E, Moiseyenko VM, Tjulandin S, Majlis A, Constenla $\mathrm{M}$, Boni C, et al. Phase III study of docetaxel and cisplatin plus fluorouracil compared with cisplatin and fluorouracil as first-line therapy for advanced gastric cancer: a report of the V325 Study Group. J Clin Oncol. 2006;24:4991-7.

9. Cunningham D, Starling N, Rao S, Iveson T, Nicolson M, Coxon $\mathrm{F}$, et al. Capecitabine and oxaliplatin for advanced esophagogastric cancer. N Engl J Med. 2008;358:36-46.

10. Kang YK, Kang WK, Shin DB, Chen J, Xiong J, Wang J, et al. Capecitabine/cisplatin versus 5-fluorouracil/cisplatin as first-line therapy in patients with advanced gastric cancer: a randomised phase III noninferiority trial. Ann Oncol. 2009;20:666-73.

11. NCCN Guidelines Version 5. Gastric cancer NCCN evidence blocks. 2017. https://www.nccn.org/professionals/physician_gls/ pdf/gastric_blocks.pdf. Accessed 4 Nov 2017.

12. Bang YJ, Van Cutsem E, Feyereislova A, Chung HC, Shen L, Sawaki A, et al. Trastuzumab in combination with chemotherapy versus chemotherapy alone for treatment of HER2-positive advanced gastric or gastro-oesophageal junction cancer (ToGA): a phase 3, open-label, randomised controlled trial. Lancet. 2010;376:687-97.

13. Ohtsu A, Shah MA, Van Cutsem E, Rha SY, Sawaki A, Park $\mathrm{SR}$, et al. Bevacizumab in combination with chemotherapy as first-line therapy in advanced gastric cancer: a randomized, 
double-blind, placebo-controlled phase III study. J Clin Oncol. 2011;29:3968-76.

14. Takiuchi H, Goto M, Imamura H, Furukawa H, Imano M, Imamoto $\mathrm{H}$, et al. Multi-center phase II study for combination therapy with paclitaxel/doxifluridine to treat advanced/recurrent gastric cancer showing resistance to S-1 (OGSG 0302). Jpn J Clin Oncol. 2008;38:176-81.

15. Ono A, Boku N, Onozawa Y, Hironaka S, Fukutomi A, Yasui $\mathrm{H}$, et al. Activity of S-1 in advanced or recurrent gastric cancer patients after failure of prior chemotherapy, including irinotecan + cisplatin or fluorouracil (except S-1). Jpn J Clin Oncol. 2009;39:332-5.

16. Sakata Y, Ohtsu A, Horikoshi N, Sugimachi K, Mitachi Y, Taguchi T. Late phase II study of novel oral fluoropyrimidine anticancer drug S-1 (1M tegafur-0.4M gimestat-1M otastat potassium) in advanced gastric cancer patients. Eur J Cancer. 1998;34:1715-20.

17. Koizumi W, Kurihara M, Nakano S, Hasegawa K. Phase II study of S-1, a novel oral derivative of 5-fluorouracil, in advanced gastric cancer. For the S-1 Cooperative Gastric Cancer Study Group. Oncology. 2000;58:191-7.

18. Miwa M, Ura M, Nishida M, Sawada N, Ishikawa T, Mori K, et al. Design of a novel oral fluoropyrimidine carbamate, capecitabine, which generates 5-fluorouracil selectively in tumours by enzymes concentrated in human liver and cancer tissue. Eur J Cancer. 1998;34:1274-81.

19. Ajani JA, Rodriguez W, Bodoky G, Moiseyenko V, Lichinitser $\mathrm{M}$, Gorbunova V, et al. Multicenter phase III comparison of cisplatin/S-1 with cisplatin/infusional fluorouracil in advanced gastric or gastroesophageal adenocarcinoma study: the FLAGS trial. J Clin Oncol. 2010;28:1547-53.

20. Yamada Y, Yamamoto S, Ohtsu A, Suzuki Y, Nasu J, Yamaguchi $\mathrm{K}$, et al. Impact of dihydropyrimidine dehydrogenase status of biopsy specimens on efficacy of irinotecan plus cisplatin, $\mathrm{S}-1$, or
5-FU as first-line treatment of advanced gastric cancer patients in JCOG9912. ASCO Meet Abstr. 2009;27(15s):4535.

21. Koizumi W, Okayasu I, Hyodo I, Sakamoto J, Kojima H. Prediction of the effect of capecitabine in gastric cancer by immunohistochemical staining of thymidine phosphorylase and dihydropyrimidine dehydrogenase. Anticancer Drugs. 2008;19:819-24.

22. Ichikawa W, Takahashi T, Suto K, Hirayama R. Gene expressions for thymidylate synthase (TS), orotate phosphoribosyltransferase (OPRT), and thymidine phosphorylase (TP), not dihydropyrimidine dehydrogenase (DPD), influence outcome of patients (pts) treated with S-1 for gastric cancer (GC). J Clin Oncol (Meet Abstr) ASCO Meet Abstr. 2004;22(14_suppl):4050.

23. Napieralski R, Ott K, Kremer M, Specht K, Vogelsang H, Becker $\mathrm{K}$, et al. Combined GADD45A and thymidine phosphorylase expression levels predict response and survival of neoadjuvanttreated gastric cancer patients. Clin Cancer Res. 2005;11:3025-31.

24. Hironaka S, Ueda S, Yasui H, Nishina T, Tsuda M, Tsumura T, et al. Randomized, open-label, phase III study comparing irinotecan with paclitaxel in patients with advanced gastric cancer without severe peritoneal metastasis after failure of prior combination chemotherapy using fluoropyrimidine plus platinum: WJOG 4007 trial. J Clin Oncol. 2013;31:4438-44.

25. Higuchi K, Tanabe S, Shimada K, Hosaka H, Sasaki E, Nakayama N, et al. Biweekly irinotecan plus cisplatin versus irinotecan alone as second-line treatment for advanced gastric cancer: a randomised phase III trial (TCOG GI-0801/BIRIP trial). Eur J Cancer. 2014;50:1437-45.

26. Ford HE, Marshall A, Bridgewater JA, Janowitz T, Coxon FY, Wadsley J, et al. Docetaxel versus active symptom control for refractory oesophagogastric adenocarcinoma (COUGAR-02): an open-label, phase 3 randomised controlled trial. Lancet Oncol. 2014;15:78-86.

\section{Affiliations}

Kazuhiro Nishikawa ${ }^{1}$ (D $\cdot$ Akira Tsuburaya $^{2} \cdot$ Takaki Yoshikawa $^{3} \cdot$ Masazumi Takahashi $^{4} \cdot$ Kazuaki Tanabe $^{5}$. Kensei Yamaguchi ${ }^{6}$. Shigefumi Yoshino ${ }^{7}$. Tsutomu Namikawa ${ }^{8} \cdot$ Toru Aoyama $^{3}$. Yasushi Rino ${ }^{9}$. Junji Kawada ${ }^{10}$. Akihito Tsuji ${ }^{11} \cdot$ Koichi Taira $^{12} \cdot$ Yutaka Kimura $^{13}$. Yasuhiro Kodera ${ }^{14} \cdot$ Yoshinori Hirashima $^{15} \cdot$ Hiroshi Yabusaki $^{16}$. Naoki Hirabayashi ${ }^{17} \cdot$ Kazumasa Fujitani $^{10} \cdot$ Yumi Miyashita $^{18} \cdot$ Satoshi Morita $^{19} \cdot$ Junichi Sakamoto $^{20}$

1 Department of Surgery, Osaka National Hospital, 2-1-14 Houenzaka, Chuo-ku, Osaka 540-0006, Japan

2 Department of Surgery, Tsuboi Cancer Center Hospital, Koriyama, Japan

3 Department of Gastrointestinal Surgery, Kanagawa Cancer Center, Yokohama, Japan

4 Department of Surgery, Yokohama Municipal Citizen's Hospital, Yokohama, Japan

5 Department of Gastroenterological and Transplant Surgery, Hiroshima University, Hiroshima, Japan

6 Division of Gastroenterology, Saitama Cancer Center, Kita-Adachi-Gun, Japan

7 Oncology Center, Yamaguchi University Hospital, Ube, Japan

8 Department of Surgery I, Kochi Medical School, Nankoku, Japan
9 Department of Surgery, Yokohama City University, Yokohama, Japan

10 Department of Surgery, Osaka General Medical Center, Osaka, Japan

11 Department of Clinical Oncology, Kobe City Medical Center General Hospital, Kobe, Japan

12 Department of Gastroenterology, Osaka City University Graduate School of Medicine, Osaka, Japan

13 Department of Surgery, Faculty of Medicine, Kindai University, Osakasayama, Japan

14 Department of Gastroenterological Surgery, Nagoya University Graduate School of Medicine, Nagoya, Japan

15 Department of Medical Oncology and Hematology, Faculty of Medicine, Oita University, Oita, Japan

16 Department of Gastroenterological Surgery, Niigata Cancer Center Hospital, Niigata, Japan 
17 Department of Surgery, Hiroshima City Asa Hospital, Hiroshima, Japan

18 Epidemiological and Clinical Research Information Network (ECRIN), Okazaki, Japan
19 Department of Biomedical Statistics and Bioinformatics, Graduate School of Medicine, Kyoto University, Kyoto, Japan

20 Tokai Central Hospital, Kakamigahara, Japan 